\title{
AVALIAÇÃO DA UTILIZAÇÃO DO PLACEBO NAS CRISES AGUDAS DE MIGRÂNEA SEM AURA, MIGRÂNEA COM AURA E CEFALÉIA DO TIPO TENSIONAL EPISÓDICA
}

\author{
Marcelo Eduardo Bigal', Janaína Oline Maciel Bigal', \\ Carlos Alberto Bordinß', José Geraldo Speciali
}

\begin{abstract}
RESUMO - O presente estudo apresenta a avaliação do efeito placebo no tratamento agudo da migrânea sem aura, migrânea com aura e cefaléia do tipo tensional episódica. Foram estudados pacientes que deram entrada entre 1 de março de 1997 a 31 de novembro de 1999 em dois pronto-socorros. Definiram-se três grupos, cada um com 30 pacientes: 1 - migrânea sem aura (MSA); 2 - migrânea com aura (MCO); 3 - cefaléia do tipo tensional episódica (CTTE). Os pacientes participavam de estudo randomizado para avaliar a eficácia de 4 drogas; aqueles randomizados para o placebo foram incluídos também. Os parâmetros avaliados foram: 1 dor; 2 - sintomas associados. Avaliados uma hora após a administração do placebo, 50\% dos pacientes do grupo MSA, 23,3\% dos pacientes do grupo MCA e 26,7\% dos pacientes do grupo CTTE apresentavam melhora da dor. A melhora, avaliada pela escala numérica da dor foi, em média, de $41,6 \%, 23,1 \%$ e $36 \%$, respectivamente. Houve também redução significativa de todos os sintomas associados. A utilização do placebo é, portanto, essencial na avaliação de drogas utilizadas no tratamento de cefaléias agudas.
\end{abstract}

PALAVRAS-CHAVE: placebo, cefaléias agudas, migrânea, cefaléia do tipo tensional.

Evaluation of placebo use in migraine without aura, migraine with aura and episodic tension-type headache acute attacks

\begin{abstract}
This study presents an evaluation of placebo response in the acute treatment of migraine with or without aura and episodic tension type headache. We studied patients admitted between March 1 ${ }^{\text {st }}, 1997$ and November $31^{\text {st }}, 1999$ in two Emergency Room Units. Three groups had been defined, each one with 30 participants: migraine without aura (MWOA), migraine with aura (MWA) and episodic tension-type headache (ETTH). Patients were participating of a randomized study to evaluate efficacy of 4 different drugs; those randomized to receive placebo were included. We evaluated pain and associated symptoms. After one hour of placebo administration, $50 \%$ of MWOA patients, $23.3 \%$ of MWA and $26.7 \%$ of ETTH had presented pain relief. The mean of this relief, evaluated by the numerical pain scale, was $41.6 \%, 23.1 \%$ and $36 \%$, respectively. Use of placebo is essential in evaluating the therapeutic role of drugs used in the treatment of acute headache.
\end{abstract}

KEY WORDS: placebo, acute headaches, migraine, tension-type headache.

Pode-se afirmar que cefaléia é sintoma tão frequente na população geral, que raro é o indivíduo que nunca a apresentou. Rasmussen et al. ${ }^{1}$ estimaram a prevalência das cefaléias ao longo da vida, em $93 \%$ dos homens e $99 \%$ das mulheres. As cefaléias mais prevalentes na população são a cefaléia do tipo tensional, com prevalência ao longo de um ano ao redor de $63 \%$ dos homens e $86 \%$ das mulheres $^{1}$ e a migrânea, com prevalência anual ao redor de $6 \%$ dos homens e $15 \%$ das mulheres ${ }^{2 ; 3}$.

As cefaléias primárias, as mais comuns na população geral, também são as causas mais frequentes de atendimento médico por cefaléia em todos os níveis de complexidade, como demonstrado por vários estudos realizados em nosso meio: 1. Estudo que avaliou 6006 pacientes atendidos em unidades básicas de saúde mostrou que a migrânea foi a principal causa de atendimentos de pacientes com cefaléia $(45,1 \%)$. A cefaléia do tipo tensional foi responsável por $7,3 \%$ dos atendimentos ${ }^{4} ; 2$. Em unidade de emergência, Zukerman et al. ${ }^{5}$ referiram que $70,7 \%$ dos casos de cefaléia tinham etiologia primária, sendo $26,5 \%$ migrânea, $7,8 \%$ cefaléia tensovascular e $29 \%$ cefaléia do tipo tensional; 3 . Estudo realizado na Unidade de Emergência do Hospital das Clínicas, em Ribeirão Preto, mostrou que $71,1 \%$ dos

\footnotetext{
${ }^{1}$ Mestre em Neurologia pela Faculdade de Medicina de Ribeirão Preto da Universidade de São Paulo, Ribeirão Preto SP, Brasil (FMRP-USP); ${ }^{2}$ Acadêmica de Psicologia, Universidade de Ribeirão Preto (UNAERP); ${ }^{3}$ Doutor em Neurologia FMRP-USP; ${ }^{4}$ Professor Associado de Neurologia FMRP-USP.
}

Recebido 2 Janeiro 2001, recebido na forma final 9 Março 2001. Aceito 15 Março 2001. 
casos de cefaléia eram de etiologia primária, sendo que migrânea respondeu por $48,2 \%$ dos casos que não necessitaram internação, enquanto cefaléia do tipo tensional foi o diagnóstico de $18,3 \%$ desses casos $^{6}$.

Assim, uma questão de fundamental importância que se impõe aos médicos assistentes, frente ao elevado número de pacientes com crises agudas de migrânea e cefaléia do tipo tensional que procuram auxílio médico, diz respeito ao tratamento abortivo das crises. Ao analisar a literatura disponível, deparamo-nos basicamente com dois problemas: 1 - a proposição de protocolos que utilizam drogas não disponíveis em unidades de saúde no Brasil; 2 - a utilização de diversos protocolos, de acordo com o autor, que utilizam drogas, esquemas e posologias diferentes, avaliados com metodologia diversa, o que dificulta sua comparação.

Um questionamento atual que se impõe à experimentação científica em geral e à terapêutica da dor em particular, diz respeito à utilização do placebo. Há, basicamente, duas correntes antagônicas quanto a esse aspecto. Um primeiro grupo que não considera ética a utilização de placebo em padecentes de dores agudas ${ }^{7}$ e um segundo grupo que considera a utilização do placebo como fundamental para a confiabilidade dos dados obtidos ${ }^{8}$. Esse aspecto metodológico adquire particular importância no Brasil, porque as principais drogas utilizadas no tratamento agudo das migrânea em outros países (compostos ergotamínicos endovenosos e triptanos) não são disponíveis no sistema público de saúde e a maioria das drogas aqui utilizadas não foram testadas contra placebo.

O presente estudo visa avaliar o efeito do placebo no tratamento agudo da dor e dos sintomas associados de pacientes com migrânea sem aura, migrânea com aura e cefaléia do tipo tensional episódica, estabelecendo um padrão para a posterior determinação real da eficácia das drogas utilizadas em nosso meio para o tratamento agudo dessas cefaléias.

\section{MÉTODO}

Foram estudados pacientes que deram entrada nos anos de 1997 a 1999 (1 de março de 1997 a 31 de novembro de 1999) em duas Unidades de Saúde: Pronto Socorro do Município de São Carlos, São Paulo e Unidade Básica Distrital de Saúde Alexandre Flemming, bairro Simioni, Ribeirão Preto, São Paulo. Trata-se de Unidades Básicas de Saúde que dispõem de pronto atendimento de urgência, com funcionamento ininterrupto.

Foram incluídos no estudo todos os pacientes que preencheram os seguintes critérios: A - Idade mínima de 18 anos completos; B - Diagnóstico de migrânea sem aura, migrânea com aura ou cefaléia do tipo tensional episódica de acordo com os critérios definidos pela Sociedade Internacional de Cefaléia ${ }^{9}$, em vigência de crise de dor; C- Concordância em participar do estudo expressa pela assinatura do Termo de Consentimento Pós-Informação aprovado pelo Comitê de Ética em Pesquisa do Hospital das Clínicas da Faculdade de Medicina de Ribeirão Preto da Universidade de São Paulo.

Os pacientes que participaram desse estudo fazem parte de outro trabalho, em andamento, randomizado, controlado por placebo, com mascaramento, que visa avaliar a eficácia de 4 drogas no tratamento agudo das cefaléias em questão.

Trabalhamos com três grupos - migrânea sem aura (MSA), migrânea com aura (MCA) e cefaléia do tipo tensional episódica (CTTE), cada um com 30 pacientes. Foram aplicadas escalas de avaliação da dor e dos sintomas associados imediatamente antes da administração da substância em estudo (Tempo 0 - T0), 30 minutos (Tempo 30 T30) e 60 minutos após a administração das mesmas (Tempo 60 - T60). Todos os pacientes que puderam ser recontactados por telefone foram reavaliados 24 horas após a administração da substância em estudo. Essa reavaliação consistiu na aplicação das mesmas escalas anteriormente aplicadas nos T0, T30 e T60.

Os pacientes eram medicados na sala de medicação das unidades de saúde. São salas sem isolamento acústico, compartilhadas por pacientes com diversos problemas agudos. Permaneciam deitados e tinham veia periférica cateterizada e mantida com soro fisiológico a $0,9 \%$ (SF $0,9 \%), 0,5 \mathrm{ml}$ (10 gotas) por minuto. A seguir, recebiam injeção de $10 \mathrm{ml}$ de SF 0,9\% por via endovenosa (placebo). A administração da substância era realizada por auxiliar de enfermagem, orientada a não dizer ou deixar transparecer a natureza da substância em questão e acompanhada pelo pesquisador (lembramos que a avaliação do placebo se deu no contexto de estudo que avaliou randomicamente 4 substâncias ativas. Assim, o paciente não sabia se estava recebendo placebo ou uma dessas substâncias). Se decorrida uma hora após a administração do placebo, o controle do quadro clínico fosse insatisfatório, administrava-se um medicamento (medicação de resgate), cuja eficácia não foi avaliada no estudo.

A intensidade dos sintomas foi avaliada da seguinte maneira:

\section{1 - Intensidade da Dor}

A - Solicitava-se ao paciente que desse uma nota variando de 0 a 10, para sua dor, frisando que o valor 0 significava "ausência de dor" e o valor 10 significava "a dor mais intensa que se possa sentir".

B - Era solicitado ao paciente que categorizasse sua dor em: intensa, moderada, leve ou ausente.

\section{2 - Intensidade dos Sintomas Associados}

O paciente descrevia cada um de seus sintomas como: 
Tabela 1. Distribuição do gênero e idade segundo o grupo estudado.

\begin{tabular}{lccccc}
\hline Grupo & $\mathrm{n} / \mathrm{N}^{*}$ & $\begin{array}{c}\text { Masculino } \\
\mathrm{n}(\%)\end{array}$ & $\begin{array}{c}\text { Feminino } \\
\mathrm{n}(\%)\end{array}$ & $\begin{array}{c}\text { Feminino } \\
\text { Masculino }\end{array}$ & $\begin{array}{c}\text { Média da idade } \\
\text { (anos) }\end{array}$ \\
\hline MSA & $21 / 30$ & $12(40 \%)$ & $18(60 \%)$ & 1,5 & 27 \\
MCA & $24 / 30$ & $7(23,3 \%)$ & $23(76,7 \%)$ & 3,3 & 28,2 \\
CTTE & $19 / 30$ & $15(50 \%)$ & $15(50 \%)$ & 1 & 37,6 \\
\hline
\end{tabular}

$\mathrm{n}^{*}$, pacientes avaliados 24 horas após a administração do placebo; $N^{*}$, total de pacientes do grupo; $n$, valor absoluto; \%, valor relativo. MAS, migrânea sem aura; MCA, migrânea com aura; CTTE, cefaléia do tipo tensional episódica.

intenso, moderado, leve ou ausente, considerados, para efeito de tabulação, como tendo valores de 3 (intenso) a 0 (ausente).

Os parâmetros utilizados para avaliação da eficácia do placebo foram os seguintes:

\section{1 - Analgesia}

A - Quantificação da intensidade da dor avaliada pela escala numérica (parâmetro 1).

B - Avaliação da Eficácia Analgésica (parâmetro 2): Considerou-se uma substância eficaz, do ponto de vista de promover analgesia, quando um indivíduo com dor fraca ou moderada ficou sem dor, nos tempos determinados, ou quando um indivíduo com dor forte passou a não apresentá-la ou apresentá-la com intensidade fraca. Assim, casos em que a dor era moderada e tornou-se fraca ou era forte e tornou-se moderada, a droga utilizada não foi considerada eficaz (Fig 1).

C-Ausência de dor (parâmetro 3): foram avaliados os pacientes que referiram ausência de dor nos tempos determinados.

D - Melhora da dor (parâmetro 4): foram considerados os pacientes que apresentaram qualquer melhora na intensidade da dor quantificada pela escala numérica, nos tempos determinados.

\section{2 - Sintomas associados}

Foram avaliados através da quantificação da intensidade do sintoma avaliada pela escala numérica (parâmetro 5). Só foi utilizado para MSA e MCA.
A análise estatística consistiu da utilização do teste não paramétrico para dados pareados de Wilcoxon, considerando o nível de significância de $\mathrm{p}$ como menor que 0,05 .

\section{RESULTADOS}

As características de cada um dos grupos estudados está exposta na Tabela 1.

\section{1 - GRUPO MSA \\ A - Analgesia}

Os valores encontrados para os parâmetros utilizados na avaliação da analgesia que se seguiu à administração do placebo estão apresentados na Tabela 2 . Os valores assinalados com o símbolo * apresentam diferença estatisticamente significativa $(p<$ $0,05)$ com aqueles apresentados antes da administração do placebo (T0). Assim, nota-se redução significativa no parâmetro 1, observada uma hora após a administração do placebo e no parâmetro 4, já perceptível 30 minutos após a administração do placebo. Para todos os parâmetros, houve diferença estatisticamente significativa na avaliação de 24 horas. Dos três pacientes que apresentavam-se sem dor no T60, dois $(66,7 \%)$ apresentaram recorrência. Quando avaliados 24 horas após a administração, 10 dos 21 pacientes $(47,6 \%)$ haviam utilizado algum analgésico para controle da dor.

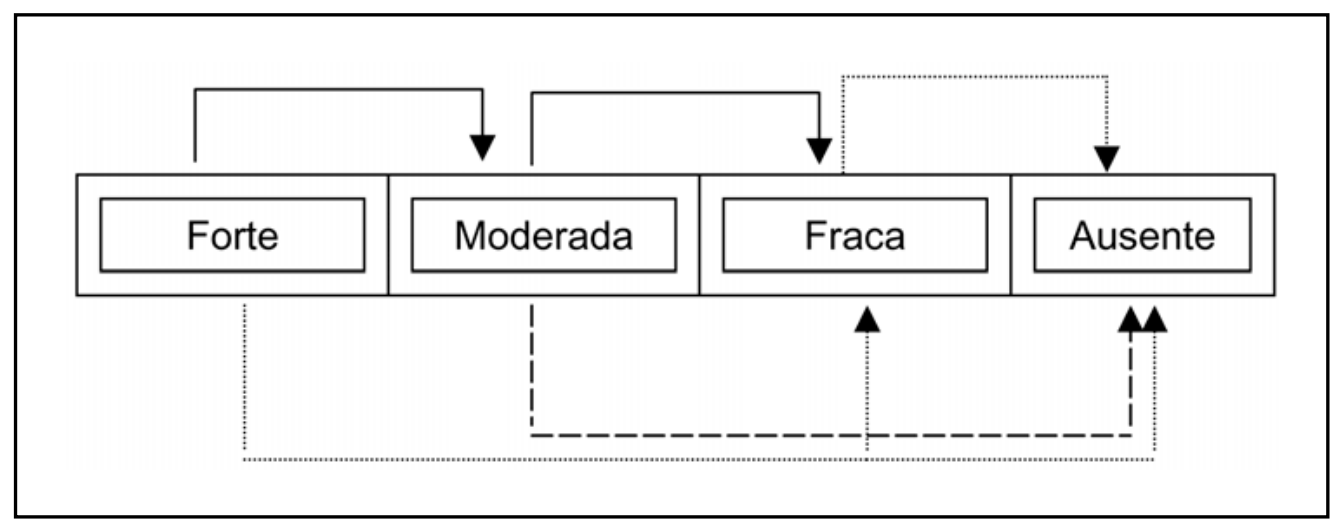

Fig 1. Critérios de eficácia analgésica considerados.

Em tracejado estão representadas as modificações da dor consideradas como critérios de eficácia. 

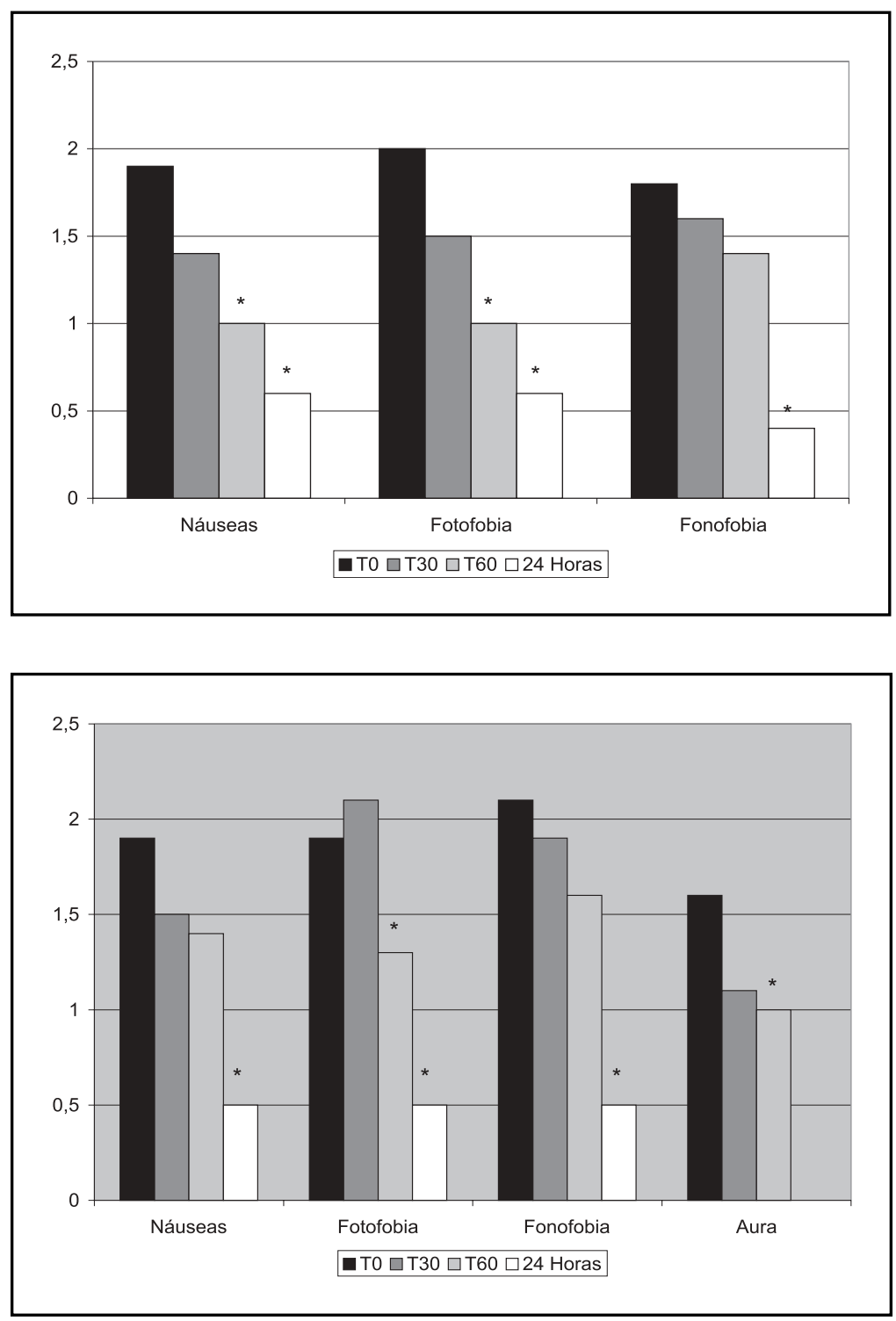

Fig 2. Quantificação dos sintomas associados pela escala numérica, do grupo migrânea sem aura.

Fig 3. Quantificação dos sintomas associados pela escala numérica, grupo migrânea com aura.

\section{B - Sintomas Associados}

$\mathrm{Na}$ avaliação inicial, dos 30 pacientes, 23 apresentavam náuseas; 27 , fotofobia; 28 , fonofobia. Foram recontactados 24 horas após, respectivamente, 15 pacientes (65,2\% em relação ao T0); 17 pacientes $(62,9 \%)$ e 23 pacientes $(82,1 \%)$. A evolução de cada um dos sintomas associados, quantificados pela escala numérica (que variou de 0 a 3 para os sintomas associados - parâmetro 5), está exposta na Figura 2. Houve diferença estatisticamente significativa, no que diz respeito à diminuição das náuseas e fotofobia, no T60 e 24 horas após a administração do placebo. Para a fonofobia só houve diferença na reavaliação de 24 horas.

\section{2 - GRUPO MCA}

A - Analgesia

Os valores encontrados para os parâmetros utili- zados na avaliação da analgesia que se seguiu à administração do placebo estão apresentados na Tabela 3. A exemplo do observado no grupo MSA, nota-se melhora estatisticamente significativa do parâmetro 4, uma hora após a administração do placebo. Para os demais parâmetros, a significância estatística foi verificada na reavaliação de 24 horas. Muito embora não tenha havido significância estatística, é importante salientar que houve melhora média da intensidade da dor aferida pela escala de dor (parâmetro 1), de 16,7\% 30 minutos após a administração do placebo, de $23,1 \%$ após 60 minutos e de $76,9 \%$ após 24 horas (esse valor mostrou significância estatística). Dos dois pacientes que apresentavam-se sem dor no T60, um apresentou recorrência. Quando avaliados 24 horas após a administração, 12 dos 24 pacientes (50\%) haviam utilizado algum analgésico para controle da dor. 
Tabela 2. Evolução dos parâmetros utilizados na avaliação da analgesia em pacientes do grupo migrânea sem aura.

\begin{tabular}{lcccc}
\hline & $\begin{array}{c}\text { Escala numérica } \\
\text { (Parâmetro 1) } \\
\text { X (DP) }\end{array}$ & $\begin{array}{c}\text { Eficácia Analgésica } \\
\text { (Parâmetro 2) } \\
\mathrm{n} / \mathrm{N}(\%)\end{array}$ & $\begin{array}{c}\text { Ausência de Dor } \\
(\text { Parâmetro 3) } \\
\mathrm{n} / \mathrm{N}(\%)\end{array}$ & $\begin{array}{c}\text { Melhora da Dor } \\
\text { (Parâmetro 4) } \\
\mathrm{n} / \mathrm{N}(\%)\end{array}$ \\
\hline T0 & $7,7(2,2)$ & $0 / 30$ & $0 / 30$ & $0 / 30$ \\
T30 & $5,9(2,4)$ & $3 / 30(10 \%)$ & $1 / 30(3,3 \%)$ & $18 / 30(60 \%)^{*}$ \\
T60 & $4,5(3,5)^{*}$ & $5 / 30(16,7 \%)$ & $3 / 30(10 \%)$ & $15 / 30(50 \%)^{*}$ \\
24 horas & $3,3(2,8)^{*}$ & $13 / 21(31,9 \%)^{*}$ & $7 / 21(33,3 \%)^{*}$ & $14 / 21(66,7 \%)^{*}$ \\
\hline
\end{tabular}

$\mathrm{X}$, média; DP, desvio padrão; $\mathrm{n}$, número de pacientes com resposta positiva; $\mathrm{N}$, número total de pacientes submetidos à droga; $\%$, percentual de resposta positiva; ${ }^{*}, p<0,05$.

Tabela 3. Evolução dos parâmetros utilizados na avaliação da analgesia em pacientes do grupo migrânea com aura.

\begin{tabular}{lcccc}
\hline & $\begin{array}{c}\text { Escala numérica } \\
\text { (Parâmetro 1) } \\
\mathrm{X}(\mathrm{DP})\end{array}$ & $\begin{array}{c}\text { Eficácia Analgésica } \\
(\text { Parâmetro 2) } \\
\mathrm{n} / \mathrm{N}(\%)\end{array}$ & $\begin{array}{c}\text { Ausência de Dor } \\
\text { (Parâmetro 3) } \\
\mathrm{n} / \mathrm{N}(\%)\end{array}$ & $\begin{array}{c}\text { Melhora da Dor } \\
\text { (Parâmetro 4) } \\
\mathrm{n} / \mathrm{N}(\%)\end{array}$ \\
\hline T0 & $7,8(2,8)$ & $0 / 30$ & $0 / 30$ & $0 / 30$ \\
T30 & $6,5(3,0)$ & $1 / 30(3,3 \%)$ & $0 / 30$ & $3 / 30(10 \%)$ \\
T60 & $6,0(3,7)$ & $4 / 30(13,3 \%)$ & $2 / 30(6,7 \%)$ & $7 / 30(23,3 \%)^{*}$ \\
24 horas & $1,8(2,3)^{*}$ & $20 / 24(83,3 \%)^{*}$ & $18 / 24(75 \%)^{*}$ & $22 / 24(91,7 \%)^{*}$ \\
\hline
\end{tabular}

$\mathrm{X}$, média; DP, desvio padrão; $\mathrm{n}$, número de pacientes com resposta positiva; $\mathrm{N}$, número total de pacientes submetidos à droga; $\%$, percentual de resposta positiva.

Tabela 4. Evolução dos parâmetros utilizados na avaliação da analgesia em pacientes do grupo cefaléia do tipo tensional episódica.

\begin{tabular}{lcccc}
\hline & $\begin{array}{c}\text { Escala numérica } \\
\text { (Parâmetro 1) } \\
\text { X (DP) }\end{array}$ & $\begin{array}{c}\text { Eficácia Analgésica } \\
\text { (Parâmetro 2) } \\
\text { n/N (\%) }\end{array}$ & $\begin{array}{c}\text { Ausência de Dor } \\
\text { (Parâmetro 3) } \\
\mathrm{n} / \mathrm{N}(\%)\end{array}$ & $\begin{array}{c}\text { Melhora da Dor } \\
\text { (Parâmetro 4) } \\
\mathrm{n} / \mathrm{N}(\%)\end{array}$ \\
\hline T0 & $6,1(2,1)$ & $0 / 30$ & $0 / 30$ & $0 / 30$ \\
T30 & $4,1(1,2)^{*}$ & $3 / 30(10 \%)$ & $2 / 30(6,7 \%)$ & $5 / 30(16,7 \%)^{*}$ \\
T60 & $4,0(1,0)^{*}$ & $4 / 30(13,3 \%)$ & $3 / 30(10 \%)$ & $8 / 30(26,7 \%)^{*}$ \\
24 horas & $2,0(1,3)^{*}$ & $13 / 19(68,4 \%)^{*}$ & $12 / 19(63,1 \%)^{*}$ & $17 / 19(89,5 \%)^{*}$ \\
\hline
\end{tabular}

$\mathrm{n}$, número de pacientes com resposta positiva; N, número total de pacientes submetidos à droga; \%, percentual de resposta positiva.

\section{$B$ - Sintomas Associados}

$\mathrm{Na}$ avaliação inicial, dos 30 pacientes, 27 apresentavam náuseas; 27 , fotofobia; 24 , fonofobia. Foram recontactados 24 horas após, respectivamente, 20 pacientes $(74,1 \%$ em relação ao T0); 21 pacientes $(77,8 \%)$ e 21 pacientes $(77,8 \%)$. A evolução de cada um dos sintomas associados, quantificados pela escala numérica, está exposta na Figura 3. Houve diferença estatisticamente significativa, no que diz respeito à diminuição das náuseas e fonofobia, 24 horas após a administração do placebo. Para a fotofobia já houve diferença no T60 e também na reavaliação de 24 horas.

\section{3 - GRUPO CTTE}

A - Analgesia

A evolução da dor aferida pela escala numérica e os demais parâmetros utilizados para avaliar a analgesia que se seguiu à administração do placebo estão apresentados na Tabela 4. Houve melhora estatisticamente significativa no parâmetro 1 , nos três momentos testados. Essa melhora foi da ordem de $33 \%$ no T30, 36\% no T60 e 65,6\% 24 horas após. O parâmetro 4 já mostrou redução estatisticamente significativa com 30 minutos e manteve-se nos demais períodos avaliados. Para os demais parâmetros avaliados (parâmetros 2 e 3), a diferença só foi significativa após 24 horas da administração do placebo.

Dos três pacientes que apresentavam-se sem dor no T60, um apresentou recorrência. Quando avaliados 24 horas após a administração, 7 dos 19 pacientes $(36,8 \%)$ haviam utilizado algum analgésico para controle da dor. 


\section{DISCUSSÃO}

Há mais de um século, Claude Bernard formalizou os critérios para a obtenção de informações válidas na medicina experimental, porém a aplicação desses critérios à terapêutica e ao processo de tomada de decisões que a envolve tem sido lento e inconsistente. Em termos históricos, a ausência de dados precisos sobre os efeitos das drogas em seres humanos se devia, em grande parte, aos padrões éticos da experimentação em humanos. Com a meIhor definição desses fatores, abre-se caminho para a experimentação clínica $^{10}$.

Coube a Sir Austin Brandford Hill, estudando o papel da estreptomicina no tratamento da tuberculose, o crédito de ter conduzido o primeiro estudo randomizado da era moderna ${ }^{11,12}$. Desde então, o número de estudos clínicos randomizados aumentou exponencialmente. A Cochrane Library listava, no ano de 1998, mais de 150.000 estudos desse tipo $^{13}$, que servem de base para a denominada Medicina Baseada em Evidências ${ }^{14}$. A metodologia que define os estudos clínicos randomizados controlados (ECRC) é uma das mais simples, poderosas e revolucionárias ferramentas de pesquisa disponíveis ${ }^{15}$. Em essência, um ECRC é aquele em que seus participantes são alocados de maneira aleatória, randomizada portanto, para receber uma de duas ou mais intervenções clínicas possíveis ${ }^{16}$. Usualmente, uma das intervenções é definida como controle, constituindo condição sine qua non em qualquer ensaio clínico. A seleção de um grupo controle apropriada é tão crítica à utilidade eventual de um experimento quanto a seleção do grupo experimental 17;18-22.

O grupo controle pode consistir de "terapias padrões" ou de placebo, podendo a escolha entre esses tipos ser determinada explicitamente por questionamentos éticos. Curran ${ }^{18}$ considera que, de modo geral, o placebo deve ser evitado em situações que envolvam risco de vida ou o aparecimento de morbidade (nessas situações deve ser utilizada alguma terapia já estabelecida para a doença em questão, como controle), devendo ser utilizado em todas as outras situações.

$O$ efeito placebo manifesta-se em qualquer ato médico, sendo, portanto, necessário para a boa condução de qualquer ensaio clínico. Estudos indicam que o efeito placebo é mais acentuado na terapêutica da dor, sendo, portanto, particularmente importante em estudos que avaliem terapêuticas analgésicas $^{23}$. Turner et al. ${ }^{24}$, conduziram meta-análise para avaliar o efeito placebo no estudo de tratamentos analgésicos concluindo que há superestimação da eficácia desses tratamentos pela não consideração correta do efeito placebo.

$O$ efeito placebo também foi considerado na pesquisa de medicamentos utilizados em cefaléias. Weissman ${ }^{25}$, em artigo de revisão, refere que o placebo propicia alívio em $25 \%$ a $35 \%$ dos pacientes com migrânea e em $55 \%$ dos pacientes com cefaléia do tipo tensional. Estudo anterior já havia considerado a importância da utilização do placebo em estudos sobre cefaléia, tendo mostrado melhora de cerca de $43 \%$ dos pacientes que receberam essa terapêutica (não especificando o tipo de cefaléia) ${ }^{26}$. DalsgaardNielsen ${ }^{27}$ considerou que havia melhora em $25 \%$ dos pacientes com cefaléia que recebiam placebo.

Nossos dados mostraram que, avaliados uma hora após a administração do placebo, $50 \%$ dos pacientes do grupo MSA (Tabela 2), 23,3\% dos pacientes do grupo MCA (Tabela 3 ) e $26,7 \%$ dos pacientes do grupo CTTE (Tabela 4) apresentavam melhora da dor (parâmetro 4). Quando avaliamos a escala da dor (parâmetro 1), observamos que houve melhora da intensidade média da dor de $23,4 \%, 41,6 \%$ e $57,1 \%$ respectivamente no T30, T60 e 24 horas após a administração, nos pacientes do grupo MSA. No grupo MCA esses valores foram, respectivamente $16,7 \%, 23,1 \%$ e $76,9 \%$ e, no grupo CTTE, 33\%, 36\% e $65,5 \%$. Esses dados são de fundamental importância. Se os valores encontrados para os pacientes do grupo MSA ( $50 \%$ dos pacientes apresentando alguma melhora em uma hora, sendo a melhora média de $41,6 \%$ ) fossem obtidos em um estudo aberto para avaliar determinada droga, poder-se-ia erroneamente concluir que essa droga tivesse boa eficácia no tratamento agudo da migrânea sem aura.

A metodologia por nós empregada permite supor que a utilização de mais de um método para aferir eficácia analgésica garante maior fidedignidade de análise. Os parâmetros 1 (escala de dor) e 3 (melhora da dor) mostraram-se mais sensíveis em detectar o efeito placebo do que os parâmetros 2 (eficácia analgésica) e 4 (ausência de dor - Tabelas 2-4).

Muito embora a questão do placebo na avaliação da analgesia do tratamento agudo das cefaléias tenha sido abordada por alguns autores, não é de nosso conhecimento nenhum estudo que avalie o efeito placebo sobre os sintomas associados da migrânea. Nossos dados mostram que esse efeito é significativo. No grupo MSA, houve melhora estatisticamente significativa das náuseas e fotofobia nos T60 e 24 horas (Fig 2). No grupo MCA, houve meIhora da fotofobia nos T60 e 24 horas (Fig 3). As náuseas e fonofobia apresentaram melhora estatis- 
ticamente significativa em 24 horas mas, muito embora esses valores não tenham mostrado significância estatística, houve melhora média de $26,3 \%$ na intensidade das náuseas e de $38 \%$ na intensidade da fotofobia no T60. A aura apresentou melhora significativa no T60, mas esse valor é de difícil interpretação, uma vez que a própria classificação da Sociedade Internacional de Cefaléia determina que a aura deva durar menos de uma hora?.

$O$ conceito de que o efeito placebo é significativo em pacientes com cefaléia persiste até os dias atuais. Tfelt-Hansen ${ }^{8}$ refere que o controle por placebo é fundamental nos estudos sobre tratamento em cefaléia. Considera que aqueles estudos que, por qualquer motivo, utilizam uma droga reconhecidamente eficaz como controle, partem de falha metodológica grave, que deve ser reconhecida na interpretação dos resultados. Julga, além disso, que sendo o placebo secundário à relação médico-paciente, não se pode considerar dados obtidos em um estudo como extrapoláveis para estudos semelhantes realizados em outro local, tese que já havia sido considerada pelo autor anteriormente. Diener et al. ${ }^{29}$ acrescentam que, além do placebo ser fundamental na confiabilidade dos dados, seu número não pode ser excessivamente pequeno, pois quanto menor o número de participantes do grupo placebo, maior a superestimação dos seus resultados.

Concluímos, da análise de nossos dados e da revisão da literatura, ser a utilização do placebo fundamental em estudos de drogas utilizadas no tratamento das cefaléias agudas. O efeito placebo é significativo não apenas na mensuração da dor, mas também dos sintomas associados da migrânea. Sendo influenciado por fatores locais e da relação médico-paciente, os valores obtidos para o placebo não podem ser extrapoláveis de um estudo para outro, mesmo se realizados em situações semelhantes. Assim, se considerarmos que, do ponto de vista ético, o menor número possível de pacientes com cefaléias agudas deva ser submetido a tratamento placebo, os estudos devem ser dirigidos para a melhor determinação desses grupos, com técnicas de amostragem mais apuradas e sofisticação do tratamento estatístico, porém sem cogitar eliminá-los. Ao contrário, sendo os estudos abertos referentes ao tratamento agudo das cefaléias tão criticáveis, poderíamos argumentar que a não utilização do placebo poderia levar à indicação de drogas não eficazes para o tratamento agudo das cefaléias. A eventual utilização de tais drogas por médicos e pacientes que erro- neamente acreditassem na sua eficácia é que, a nosso ver, deve ser considerada como desvio ético sério.

\section{BIBLIOGRAFIA}

1. Rasmussen BK, Jansen R, Olesen J. A population-based analysis of the diagnostic criteria of the International Headache Society. Cephalalgia 1991;11: 129-134.

2. Rasmussen BK. Epidemiology of headache. Cephalalgia 1995;15:45-68.

3. Stewart WF, Lipton RB, Celentano DD, Reed ML. Prevalence of migraine headache in the United States. JAMA 1992;267:64-69.

4. Bigal ME, Bordini CA, Speciali JG. Etiology and distribution of headaches in two brazilian primary care units. Headache 2000;40:241247.

5. Zukerman E, Lima JGC, Hannuch SNM. Unidade de atendimento de agudos com cefaléia (UAAC): uma experiência nova em nosso meio. Rev Ass Med Bras 1989;35:107-110.

6. Bigal ME. Cefaléia em uma unidade de emergência de atendimento terciário. Aspectos epidemiológicos, clínicos, terapêuticos e dos custos do atendimento hospitalar. Dissertação de mestrado, FMRP-USP. Ribeirão Preto, 2000.

7. Blau JN. In: Blau JN. Migraine: clinical, therapeutic, conceptual and research aspects. London: Chapman and Hall, 1987:676-677.

8. Tfelt-Hansen P. How to define the best efficacy parameters for migraine. Cephalalgia 1997;17(Suppl 4):6-9.

9. Headache Classification Committee of the International Headache Society. Classification and diagnostic criteria for headache disorders cranial neuralgia and facial pain. Cephalalgia 1988;8(Suppl 7):1-96.

10. Blaschke TF, Nies AS, Mamelok RD. Princípios Da Terapêutica. In Gilman AF, Goodman LS, Rall TW, Murad F (eds). As Bases Farmacológicas da Terapêutica. Rio de Janeiro: Guanabara 1987:32-41.

11. Daniels M, Hill AB. Chemotherapy of pulmonary tuberculosis in young adult: an analysis of the combined results of three medical research council trials. Br Med J 1952;1:1162-1168.

12. Hill AB. The clinical trial. New Eng J Med 1952;247:113-119.

13. The Cochrane Library. Oxford: update software Issue 1, 1998.

14. Evidence-Based Medicine Working Group. Evidence-based medicine: a new approach to teaching the practice of medicine. JAMA 1992;268: 2420-2425

15. Silverman WA, Chalmers I. Sir Austin Bradford Hill: an appreciation. Controlled Clin Trials 1992;13:100-105.

16. Jadad AR, Rennie D. The randomized controlled trials gets a middleaged checkup. JAMA 1998;279:319-320.

17. Feinstein AR. Clinical biostatistics. Hard science, soft data and the challenges of choosing clinical variables in research. Clin Pharmacol Ther 1977;22:45-48.

18. Curran WJ. Reasonableness and randomization in clinical trials: fundamental law and governnmental regulation. N Engl J Med 1979;300:1273-1275.

19. Benson H, Mccallie DP. Angina pectoris and the placebo effect. N Engl J Med 1979;300:1424-1425.

20. Godfroid IO. Placebo. 1. Placebo effect - the fly in the ointment. Ann Med Psychol 1997;155:436-443.

21. Vrhovac B. Placebo and its importance in medicine. Int J Clin Pharmacol Biopharm 1977;15:161-165.

22. Bourne HR. Rational use of placebo. In Melmon KL, Morreli HF. Clinical Pharmacology: Basic Principles in Therapeutics. New York: Macmillan 1978:1052-1062.

23. Wall PD. Pain and the placebo response. Ciba Found Symp 1993;174: 187-211.

24. Turner JA, Deyo RA, Loeser JD. The importance of placebo effects in pain treatment and research. JAMA 1994;271:1609-1614.

25. Weissman, L. Experimental design in headache treatment. Headache 1971;10: 165-170.

26. Frey GH. The role of placebo response in clinical headache evaluations. Headache 1961;1:31-38.

27. Dalsgaard-Nielsen T. Experiences in clinical studies of headache: headache-psyche-placebo. Headache 1964;4:236-241.

28. Olesen J, Krabbe AE, Tfelt-Hansen P. Methodological aspects of prophylactic drug trials in migraine. Cephalalgia 1981;11:127-141.

29. Diener HC, Dowson AJ, Ferrari M. Unbalanced randomization influences placebo response: scientific versus ethical issues around the uso of placebo in migraine trials. Cephalalgia 1999;19:699-700. 